\title{
Bound for the 2-Page Fixed Linear Crossing Number of Hypercube Graph via SDP Relaxation
}

\author{
A. Suebsriwichai and T. Mouktonglang \\ Department of Mathematics, Faculty of Science, Chiang Mai University, Chiang Mai 50200, Thailand \\ Correspondence should be addressed to T. Mouktonglang; thanasak.m@cmu.ac.th
}

Received 5 January 2017; Revised 20 March 2017; Accepted 10 April 2017; Published 8 May 2017

Academic Editor: Heping Zhang

Copyright (c) 2017 A. Suebsriwichai and T. Mouktonglang. This is an open access article distributed under the Creative Commons Attribution License, which permits unrestricted use, distribution, and reproduction in any medium, provided the original work is properly cited.

\begin{abstract}
The crossing number of graph $G$ is the minimum number of edges crossing in any drawing of $G$ in a plane. In this paper we describe a method of finding the bound of 2-page fixed linear crossing number of $G$. We consider a conflict graph $G^{\prime}$ of $G$. Then, instead of minimizing the crossing number of $G$, we show that it is equivalent to maximize the weight of a cut of $G^{\prime}$. We formulate the original problem into the MAXCUT problem. We consider a semidefinite relaxation of the MAXCUT problem. An example of a case where $G$ is hypercube is explicitly shown to obtain an upper bound. The numerical results confirm the effectiveness of the approximation.
\end{abstract}

\section{Introduction}

Let $G$ be a simple connected graph with a vertex-set $V(G)=$ $\left\{v_{1}, v_{2}, v_{3}, \ldots, v_{n}\right\}$ and an edge-set $E(G)=\left\{e_{1}, e_{2}, e_{3}, \ldots, e_{m}\right\}$. The crossing number of graph $G$, denoted $\operatorname{cr}(G)$, is the minimum number of pairwise intersections of edge crossing on the plane drawing of graph $G$. Clearly, $\operatorname{cr}(G)=0$ if and only if $G$ is planar. It is known that the exact crossing numbers of any graphs are very difficult to compute. In 1973, Erdös and Guy [1] wrote, "Almost all questions that one can ask about crossing numbers remain unsolved." In fact, Garey and Johnson [2] prove that computing the crossing number is NPcomplete.

A 2-page drawing of $G$ is a representation of $G$ on the plane such that its vertices are placed on a straight horizontal line $L$ according to fixed vertex ordering and its edges are drawn as a semicircle above or below $L$ but never cross $L$.

The $n$-cube or $n$-dimensional hypercube $Q_{n}$ is recursively defined in terms of the Cartesian products. The onedimension cube $Q_{1}$ is simply $K_{2}$ where $K_{2}$ is a complete graph with 2 vertices. For $n \geq 2, Q_{n}$ is defined recursively as $Q_{n-1} \times K_{2}$. The order of $Q_{n}$ is $\left|V\left(Q_{n}\right)\right|=2^{n}$ and its size is $\left|E\left(Q_{n}\right)\right|=n 2^{n-1}$. Since $Q_{n}$ is planar for $n=1,2,3$, so $\operatorname{cr}\left(Q_{n}\right)=0$ for each such $n$. Eggleton and Guy [3] showed that $\operatorname{cr}\left(Q_{4}\right)=8$ but $\operatorname{cr}\left(Q_{n}\right)$ is unknown for $n \geq 5$.
It was declared by Eggleton and Guy [3] that the crossing numbers of the hypercube $Q_{n}$ (non-2-page) for $n \geq 3$ was

$$
\operatorname{cr}\left(Q_{n}\right) \leq \frac{5}{32} 4^{n}-\left\lfloor\frac{n^{2}+1}{2}\right\rfloor 2^{n-2} .
$$

Then, in 1973, Erdös and Guy [1] conjectured equality in (1). In 1993, a lower bound of $\operatorname{cr}\left(Q_{n}\right)$ was proved by Sýkora and Vrto [4]:

$$
\operatorname{cr}\left(Q_{n}\right) \geq \frac{1}{20} 4^{n}+O\left(n^{2} 2^{n}\right) .
$$

In 2008, Faria et al. [5] constructed a new drawing of $Q_{n}$ in the plane which led to the conjectured number of crossings

$$
\frac{5}{32} 4^{n}-\left\lfloor\frac{n^{2}+1}{2}\right\rfloor 2^{n-2} .
$$

To the best of our knowledge, the fixed linear crossing number for $Q_{n}$ has not been established. In this paper, we discuss a method to obtain an approximation for fixed linear crossing number for hypercube graph.

\section{2-Page Drawings of Hypercube Graph $Q_{n}$}

Throughout this paper, we consider the ordering of hypercube graph $Q_{n}$. Since $Q_{n}$ is defined recursively as $Q_{n-1} \times K_{2}$, for 


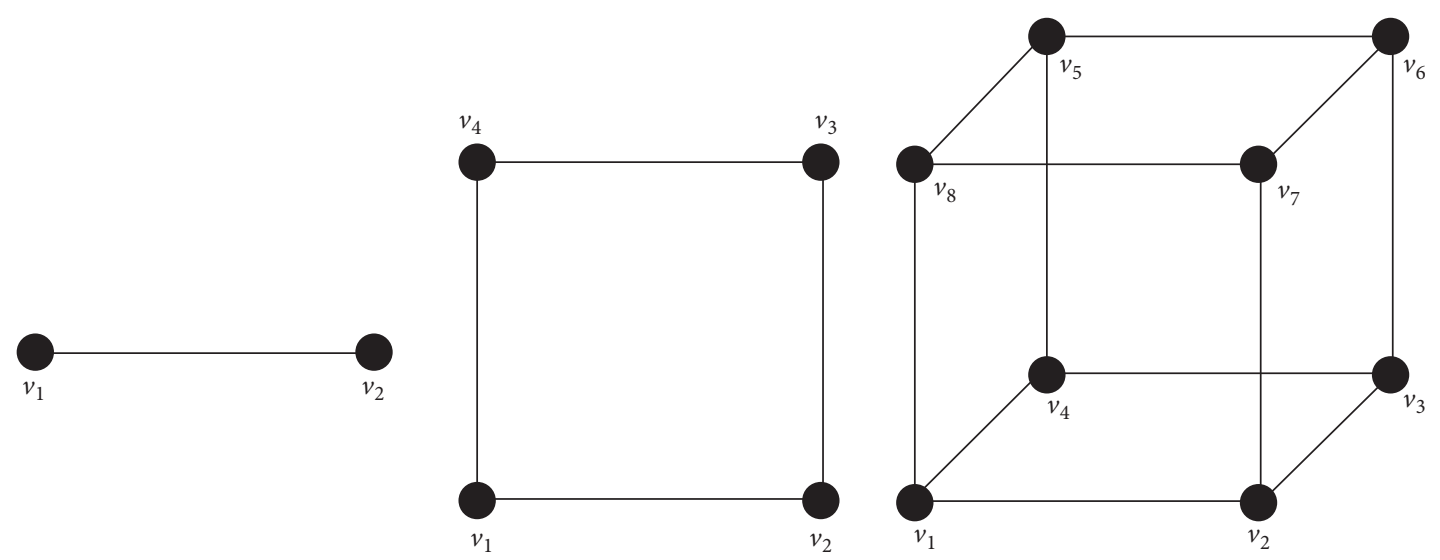

FIGURE 1: $n$-Cube graphs with fixed vertex ordering for $n=1,2,3$.

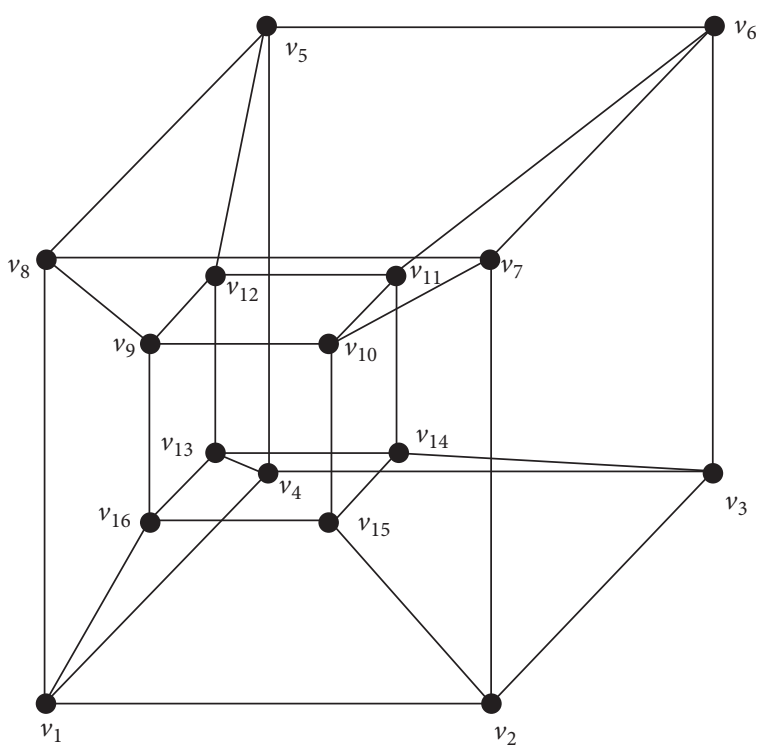

FIGURE 2: $n$-Cube graph with fixed vertex ordering for $n=4$.

$n=2, \ldots$, where $Q_{1}$ is a simple graph with 2 vertices together with a single edge incident to both vertices, $Q_{n}$ has 2 copies of $Q_{n-1}$ with edges connecting between them. Given a fixed ordering on $Q_{n-1}$, the vertices of the first $Q_{n-1}$ are labeled $1,2,3, \ldots, 2^{n-1}$ and the vertices of the second $Q_{n-1}$ are labeled $2^{n-1}+1,2^{n-1}+2, \ldots, 2^{n-1}+2^{n-1}=2^{n}$. The two vertices between the first $Q_{n-1}$ and the second $Q_{n-1}$ are adjacent if and only if the sum of the labeled is $2^{n}+1$. Figures 1 and 2 present the ordering of $Q_{1}, Q_{2}, Q_{3}$ and $Q_{4}$ which we consider throughout this paper. Notice that our method is independent on vertex ordering; therefore, for a fixed $n$, we can apply the method $\left(2^{n}\right)$ ! times so as to obtain the 2-page linear crossing number.

The 2-page drawing of $Q_{n}$ can be represented by drawing the vertices of $Q_{n}$ on a straight horizontal line $L$ according a fixed vertex ordering. Each edge fully contained one of the two half-planes (pages) as a semicircle and never cross $L$. Notice that no edge crosses itself, no adjacent edges cross each

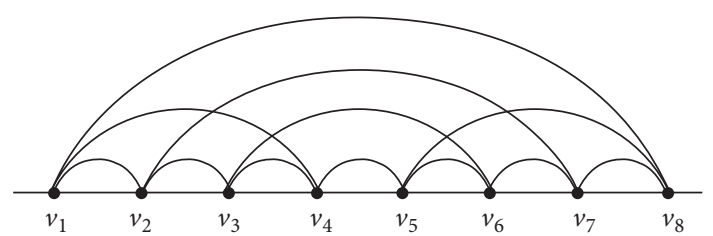

FIgURE 3: The 2-page drawing of $Q_{3}$ with fixed vertex ordering.

other, no two edges cross more than once, and no three edges cross in a point.

For a given 2-page drawing of $Q_{n}$ with the fixed vertex ordering, a pair of edges $e_{i j}=\left(v_{i}, v_{j}\right)$ and $e_{l k}=\left(v_{l}, v_{k}\right)$ are potential crossing if $e_{i j}$ and $e_{l k}$ cross each other when routed on the same side of $L$. Clearly, $e_{i j}$ and $e_{l k}$ are potential crossing if and only if $v_{i}<v_{l}<v_{j}<v_{k}$ or $v_{l}<v_{i}<v_{k}<v_{j}$.

Next we give the definition of conflict graph $G^{\prime}$ of graph $G$.

Definition 1. Given a graph $G$. We define an associated conflict graph $G^{\prime}=\left(V^{\prime}, E^{\prime}\right)$ of a graph $G=(V, E)$. There is corresponding one-to-one and onto mapping between the set of $V^{\prime}\left(G^{\prime}\right)$ and $E(G)$. Two vertices of $G^{\prime}$ are adjacent if any two edges in $G$ are potential crossing.

For example, according to the given fixed vertex ordering of $Q_{3}$ (see Figure 3), $Q_{3}^{\prime}$ is a graph of $n 2^{n-1}$ nodes, $V^{\prime}\left(Q_{3}^{\prime}\right)=$ $\left\{v_{12}^{\prime}, v_{23}^{\prime}, v_{34}^{\prime}, v_{45}^{\prime}, v_{56}^{\prime}, v_{67}^{\prime}, v_{78}^{\prime}, v_{14}^{\prime}, v_{36}^{\prime}, v_{58}^{\prime}, v_{27}^{\prime}, v_{18}^{\prime}\right\} . v_{24}^{\prime}$ and $v_{35}^{\prime}$ are adjacent in $Q_{3}^{\prime}$ because $e_{24}$ and $e_{35}$ are potential crossing in a 2-page drawing of $Q_{3}$. A fixed vertex ordering of $Q_{4}$ and its potential crossing can be seen in Figure 4.

In this paper, we are interested only in fixed linear embeddings of $Q_{n}$. There is a crossing between $e_{i j}$ and $e_{l k}$ if and only if $e_{i j}$ and $e_{l k}$ are potential crossing and embedded on the same side of $L$. We can see that the number of edge crossings depends on the order of vertices and on the sides to which the edges are assigned.

The 2-page linear crossing number of $Q_{n}$, denoted by $v_{2}\left(Q_{n}\right)$, is the minimum number of pairwise intersections of edges crossings determined by a 2-page drawing of $Q_{n}$. The 2-page fixed linear crossing number of $Q_{n}$ is the minimum 


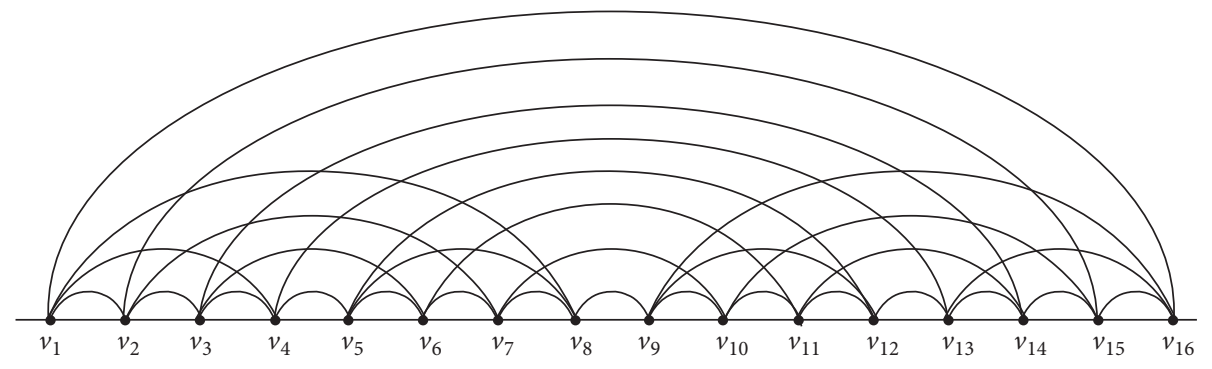

FIGURE 4: The 2-page drawing of $Q_{4}$ with fixed vertex ordering.

number of pairwise intersections of edges crossings determined by a 2-page drawing of $Q_{n}$ with fixed vertex ordering of $Q_{n}$. It is known that $v_{2}\left(Q_{n}\right)=0$ for $n=1,2,3, v_{2}\left(Q_{n}\right)>0$ for $n \geq 4$.

\section{Reduction to MAXCUT Problem}

In this section, we show that the problem can be reduced to the maximum cut problem. Next, we reduce the fixed linear crossing number problem to the maximum cut problem (MAXCUT). The MAXCUT problem is as follows.

Maximum Cut Problem (MAXCUT). Given an undirected graph $G^{\prime}=\left(V^{\prime}, E^{\prime}\right)$ the edge $e_{i j}$ of the graph is associated with nonnegative weights $a_{i j}$. The problem is to find a cut of the largest possible weight, that is, to partition the set of $V^{\prime}$ into disjoint sets $V_{1}$ and $V_{2}$ such that the total weight of all edges linking $V_{1}$ and $V_{2}$ (i.e., with one incident node in $V_{1}$ and the other one in $V_{2}$ ) is as large as possible.

In the MAXCUT problem, we may assume that the weights $a_{i j}=a_{j i} \geq 0$ are defined for every pair $i, j$ of indices: it suffices to set $a_{i j}=0$ for pairs $i, j$ of nonadjacent nodes. For the unweighted graph, we assume that $a_{i j}=1$ for $i, j=$ $1,2, \ldots, n$.

Let $G$ be a graph with a fixed vertex permutation. Given a vertex partition $\left(V_{1}, V_{2}\right)$ of its conflict graph $G^{\prime}$, the associated cut embedding is the fixed linear embedding of $G$ where edges corresponding to $V_{1}$ and $V_{2}$ are embedded to the half spaces above and below the vertex line, respectively.

Lemma 2 (see [6]).

$$
\nu_{2}(G)=\left|E^{\prime}\right|-\operatorname{MC}\left(G^{\prime}\right),
$$

where $\left|E^{\prime}\right|$ is a number of potential crossing of 2-page drawing of $G$, which is the number of edges of $G^{\prime} . M C\left(G^{\prime}\right)$ is the size of the maxcut of $G^{\prime}$.

Proof. Given a 2-page (circle) drawing of $G$, define $W \subset V_{n}$ as the chords that are drawn inside the circle. The edges of $E^{\prime}$ with precisely one endpoint in $W$ now correspond to edges of $G$ that do not cross in the drawing.

Theorem 3 (see [7]). Consider a partition $\left(V_{1}, V_{2}\right)$ of $V^{\prime}$. Then the corresponding cut embedding is a fixed linear embedding of $G$ with a minimum number of crossings if and only if $\left(V_{1}, V_{2}\right)$ is a maximum cut in $G^{\prime}$.
Proof. Let $F^{\prime}$ be the set of edges in $G^{\prime}$ with one endpoint in $V_{1}$ and one endpoint in $V_{2}$, that is, the cut given by $\left(V_{1}, V_{2}\right)$. By definition of $G^{\prime}$, we know that every crossing in the cut embedding associated with $\left(V_{1}, V_{2}\right)$ corresponds to an edge in $G^{\prime}$ such that either both its endpoint belong to $V_{1}$ or both belong to $V_{2}$, that is, to an edge in $E^{\prime} \backslash F^{\prime}$. Thus, the number of crossings is $\left|E^{\prime}\right|-\left|F^{\prime}\right|$. As $\left|E^{\prime}\right|$ is constant for a fixed vertex permutation, the result follows.

Theorem 3 reduces the fixed linear crossing number problem to the maximum cut problem (MAXCUT). In the next section, we describe the relaxation of the MAXCUT problem which leads to semidefinite programming.

3.1. Formulating MAXCUT by Semidefinite Relaxation. In this section, we show that 2-page crossing number of hypercube graph problem can be obtained by computing a semidefinite relaxation of MAXCUT.

First of all, we introduce the adjacency matrix of $G$ denoted $\operatorname{Adj}(G)$ as we know it is an $n \times n$ matrix with the property

$$
\begin{aligned}
& \operatorname{Adj}(G) \equiv\left[a_{i j}\right], \\
& a_{i j} \in\{0,1\}, a_{i j}= \begin{cases}1, & \text { if } v_{i} \text { and } v_{j} \text { are adjacent; } \\
0, & \text { otherwise }\end{cases}
\end{aligned}
$$

From $\operatorname{Adj}(G)$ we construct the conflict graph of $G$ denoted $G^{\prime}$. Finally, we perform MAXCUT on graph $G^{\prime}$. We use semidefinite relaxation to approximate the optimal value solution to the MAXCUT problem. Obviously the approximation is larger than the actual MAXCUT optimal value. The feasibility of the relaxation set is strictly larger than the original ones.

According to [2], the MAXCUT problem can be formulated as follows:

$$
\begin{array}{ll}
\max & \frac{1}{4} \sum_{i, j=1}^{n} a_{i j}\left(1-x_{i} x_{j}\right) \\
\text { s.t. } & x_{i}^{2}=1 \\
& i=1, \ldots, n .
\end{array}
$$




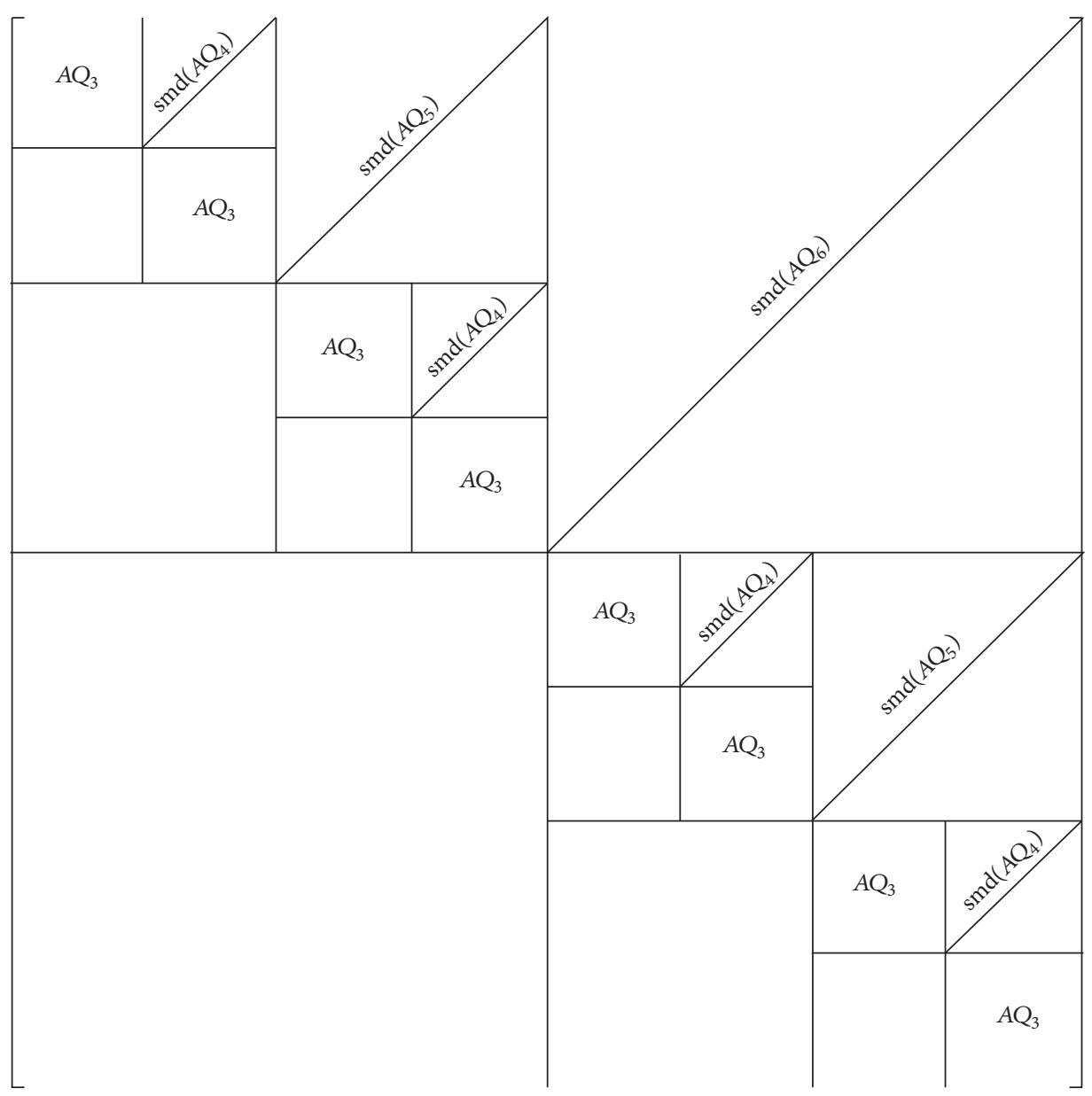

Figure 5: The adjacency matrix of size $2^{6} \times 2^{6}$ of $Q_{6}$.

We call the optimal value of (6) as "OPT." Then, the relaxation of (6) can be rewritten as

$$
\begin{array}{ll}
\max & \frac{1}{4} \sum_{i, j=1}^{n} a_{i j}\left(1-X_{i j}\right) \\
\text { s.t. } & X=\left[X_{i j}\right]_{i, j=1}^{n}=X^{T} \geq 0, \\
& X_{i i}=1, \quad i=1, \ldots, n,
\end{array}
$$

where $A=\left[a_{i j}\right]$ is an adjacency matrix of $G^{\prime}$ and $X=\left[X_{i j}\right]$ is a feasible solution to the semidefinite relaxation. The problem (7) is equivalent to

$$
\begin{array}{ll}
\min & \frac{1}{4} \operatorname{tr}(A X) \\
\text { s.t. } & X=\left[X_{i j}\right]_{i, j=1}^{n}=X^{T} \succeq 0, \\
& X_{i i}=1, \quad i=1, \ldots, n,
\end{array}
$$

where $A$ is a given adjacency matrix of $G^{\prime}$ and $X=\left[X_{i j}\right]$ is a feasible solution to the semidefinite relaxation. We call the optimal value of (8) as "SDP."
As we have seen from the relation (4), we let $\left|E_{n}^{\prime}\right|$ be the number of potential crossing of 2-page drawing of $Q_{n}$ with our fixed vertex ordering (i.e., $\left|E_{n}^{\prime}\right|$ is the number of edges of $\left.Q_{n}^{\prime}\right)$.

We can determine $\left|E_{n}^{\prime}\right|$ by considering the upper half of the main diagonal of the adjacency matrix of $Q_{n}$.

Definition 4. Let $A G=\left[a_{i j}\right]$ be the $n \times n$ adjacency matrix of $G$. The element $a_{i j}$ where $i+j=n+1$ is called minor diagonal of adjacency matrix of $G$ and the element $a_{i j}$ where $i+j=n+1, i<j$ is called semiminor diagonal of adjacency matrix of $G$, denoted by $\operatorname{smd}(A G)$.

For simplicity, the size of $\operatorname{smd}(A G)$ is a number of elements in $\operatorname{smd}(A G)$. Let $A Q_{n}$ be the adjacency matrix of graph $Q_{n}$. Therefore $A Q_{n}$ is $2^{n} \times 2^{n}$ symmetric matrix. It is clear that the size of $\operatorname{smd}\left(A Q_{n}\right)$ is $2^{n-1}$.

Let $A G_{1}$ and $A G_{2}$ be adjacency matrices of graphs $G_{1}$ and $G_{2}$, respectively; we say that the number of potential crossing between $A G_{1}$ and $A G_{2}$, denoted by PC $\left[A G_{1}, A G_{2}\right]$, is simply the number of potential crossing between 2-page drawing of graph $G_{1}$ and $G_{2}$. The adjacency matrix of size $2^{6} \times 2^{6}$ of $Q_{6}$ with respect to our ordering is presented in Figure 5. 
Lemma 5. For any integer $n \geq 5$,

$$
\begin{aligned}
\operatorname{PC} & {\left[\operatorname{smd}\left(A Q_{n-1}\right), \operatorname{smd}\left(A Q_{n}\right)\right] } \\
& =\operatorname{PC}\left[\operatorname{smd}\left(A Q_{n-1}\right), \operatorname{smd}\left(A Q_{n+i}\right)\right],
\end{aligned}
$$

where $i=1,2, \ldots, n$.

Lemma 6. For every adjacency matrix of $Q_{n}, A Q_{n}$, where $n \geq 4$, there exists adjacency matrix of $Q_{3}, A Q_{3}$, which is a submatrix embedding in $A Q_{n}$. The number of submatrix $A Q_{3}$ embedding in $A Q_{n}$ is $2^{n-3}$.

Lemma 7. For any integer $n \geq 5$,

$$
\operatorname{PC}\left[A Q_{3}, \operatorname{smd}\left(A Q_{n}\right)\right]=\operatorname{PC}\left[A Q_{3}, \operatorname{smd}\left(A Q_{n+i}\right)\right] \text {, }
$$

where $i=1,2, \ldots, n$.

Lemma 8. For any integer $n \geq 5$,

$$
\begin{aligned}
\operatorname{PC} & {\left[A Q_{n-1}, \operatorname{smd}\left(A Q_{n}\right)\right] } \\
& =\operatorname{PC}\left[A Q_{n-1}, \operatorname{smd}\left(A Q_{n+i}\right)\right],
\end{aligned}
$$

where $i=1,2, \ldots, n$.

Lemma 9. For $n \geq 4$, the number of block $\operatorname{smd}\left(A Q_{i}\right)$ embedding in $A Q_{n}$ equals $2^{n-i}, 4 \leq i \leq n$.

Lemmas 5-9 follow directly from the definitions.

Lemma 10. For any integer $n \geq 4$,

$$
\operatorname{PC}\left[\operatorname{smd}\left(A Q_{n}\right), \operatorname{smd}\left(A Q_{n+1}\right)\right]=\left(\frac{2^{n-1}}{2}\right)\left(2^{n}-2\right) \text {. }
$$

Proof. The number of potential crossing between $\operatorname{smd}\left(A Q_{n}\right)$ and $\operatorname{smd}\left(A Q_{n+1}\right)$ is a result of the number of potential crossing between all of the element $a_{1,2^{n}}, a_{2,2^{n}-1}, \ldots, a_{2^{n-1}, 2^{n-1}+1}$ in $A Q_{n}$ and $\operatorname{smd}\left(A Q_{n+1}\right)$. That is,

$$
\begin{aligned}
\operatorname{PC} & {\left[\operatorname{smd}\left(A Q_{n}\right), \operatorname{smd}\left(A Q_{n+1}\right)\right] } \\
& =\left(2^{n}-2\right)+\left(2^{n}-4\right)+\cdots+2+0 \\
& =\left(\frac{2^{n-1}}{2}\right)\left(2^{n}-2\right) .
\end{aligned}
$$

Theorem 11. For any integer $n \geq 5$,

$$
\begin{aligned}
& \left|E_{n}^{\prime}\right|=2\left|E_{n-1}^{\prime}\right|+2^{n+1}+2 \sum_{k=5}^{n}\left(2^{n-k}\right)\left(\frac{2^{k}}{4}-1\right)\left(\frac{2^{k}}{4}\right), \\
& \left|E_{4}^{\prime}\right|=40
\end{aligned}
$$

where $\left|E_{n}^{\prime}\right|$ is the number of potential crossing of 2-page drawing of $Q_{n}$ with our fixed vertex ordering.
Proof. We prove this lemma by considering the number of potential crossing of 2-page drawing of $Q_{n}$ with our fixed vertex ordering. Since $Q_{n}$ has 2 copies of $Q_{n-1}$ with some edges connecting between them, the number of potential crossing of $A Q_{n}$ is a result of twice of the number of potential crossing within $A Q_{n-1}$ together with $\operatorname{PC}\left[A Q_{3}, \operatorname{smd}\left(A Q_{n}\right)\right]$ and $\operatorname{PC}\left[\operatorname{smd}\left(A Q_{i}\right), \operatorname{smd}\left(A Q_{n}\right)\right], i=4,5, \ldots, n-1$.

Hence, it is enough to show that the number of potential crossing between 2-page drawing of $Q_{n-1}$ and $Q_{n-1}$ is equal to $\gamma(n)$, where

$$
\gamma(n)=2^{n+1}+2 \sum_{k=5}^{n}\left(2^{n-k}\right)\left(\frac{2^{k}}{4}-1\right)\left(\frac{2^{k}}{4}\right) .
$$

Note $\gamma(n)$ is the number of potential crossing between all of submatrices $A Q_{3}$ and $\operatorname{smd}\left(A Q_{n}\right)$ and also between $\operatorname{smd}\left(A Q_{i}\right)$ and $\operatorname{smd}\left(A Q_{n}\right), i=4,5, \ldots, n-1$. By Lemmas 6 and 9 ,

$$
\begin{aligned}
\gamma(n)= & 2^{n-3} \cdot \operatorname{PC}\left[A Q_{3}, \operatorname{smd}\left(A Q_{n}\right)\right]+2^{n-4} \\
& \cdot \operatorname{PC}\left[\operatorname{smd}\left(A Q_{4}\right), \operatorname{smd}\left(A Q_{n}\right)\right]+2^{n-5} \\
& \cdot \operatorname{PC}\left[\operatorname{smd}\left(A Q_{5}\right), \operatorname{smd}\left(A Q_{n}\right)\right]+\cdots \\
& +2^{n-(n-2)} \cdot \operatorname{PC}\left[\operatorname{smd}\left(A Q_{n-2}\right), \operatorname{smd}\left(A Q_{n}\right)\right] \\
& +2^{n-(n-1)} \cdot \operatorname{PC}\left[\operatorname{smd}\left(A Q_{n-1}\right), \operatorname{smd}\left(A Q_{n}\right)\right] .
\end{aligned}
$$

We precede by mathematical induction on $n$. For $n=5$, it can be easily seen that $\gamma(5)=176$ by counting. Assuming (15) holds true, now we consider $\gamma(n+1)$ as a number of potential crossing between all of the submatrices $A Q_{3}$ and $\operatorname{smd}\left(A Q_{n+1}\right)$ and also between $\operatorname{smd}\left(A Q_{i}\right)$ and $\operatorname{smd}\left(A Q_{n+1}\right), i=4,5, \ldots, n$. By the Lemmas 5, 6, 7, 9, 8, and 10,

$$
\begin{aligned}
\gamma & n+1)=2^{(n+1)-3} \cdot \mathrm{PC}\left[A Q_{3}, \operatorname{smd}\left(A Q_{n+1}\right)\right] \\
& +2^{(n+1)-4} \cdot \mathrm{PC}\left[\operatorname{smd}\left(A Q_{4}\right), \operatorname{smd}\left(A Q_{n+1}\right)\right] \\
& +2^{(n+1)-5} \cdot \mathrm{PC}\left[\operatorname{smd}\left(A Q_{5}\right), \operatorname{smd}\left(A Q_{n+1}\right)\right]+\cdots \\
& +2^{(n+1)-(n-2)} \cdot \mathrm{PC}\left[\operatorname{smd}\left(A Q_{n-2}\right), \operatorname{smd}\left(A Q_{n+1}\right)\right] \\
& +2^{(n+1)-(n-1)} \cdot \mathrm{PC}\left[\operatorname{smd}\left(A Q_{n-1}\right), \operatorname{smd}\left(A Q_{n+1}\right)\right] \\
& +2^{(n+1)-n} \cdot \mathrm{PC}\left[\operatorname{smd}\left(A Q_{n}\right), \operatorname{smd}\left(A Q_{n+1}\right)\right] \\
& =2\left(2^{n-3} \cdot \mathrm{PC}\left[A Q_{3}, \operatorname{smd}\left(A Q_{n}\right)\right]+2^{n-4}\right. \\
& \cdot \mathrm{PC}\left[\operatorname{smd}\left(A Q_{4}\right), \operatorname{smd}\left(A Q_{n}\right)\right]+2^{n-5} \\
& \cdot \mathrm{PC}\left[\operatorname{smd}\left(A Q_{5}\right), \operatorname{smd}\left(A Q_{n}\right)\right]+\cdots+2^{n-(n-2)} \\
& \cdot \mathrm{PC}\left[\operatorname{smd}\left(A Q_{n-2}\right), \operatorname{smd}\left(A Q_{n}\right)\right]+2^{n-(n-1)} \\
& \left.\cdot \mathrm{PC}\left[\operatorname{smd}\left(A Q_{n-1}\right), \operatorname{smd}\left(A Q_{n}\right)\right]\right)+2^{(n+1)-n} \\
& \cdot \mathrm{PC}\left[\operatorname{smd}\left(A Q_{n}\right), \operatorname{smd}\left(A Q_{n+1}\right)\right]=2 \gamma(n)
\end{aligned}
$$




$$
\begin{aligned}
& +2^{(n+1)-n} \cdot \operatorname{PC}\left[\operatorname{smd}\left(A Q_{n}\right), \operatorname{smd}\left(A Q_{n+1}\right)\right] \\
& =2 \gamma(n)+2^{(n+1)-n} \cdot\left(\frac{2^{n-1}}{2}\right)\left(2^{n}-2\right)=2 \gamma(n) \\
& +(2)\left(2^{n-1}\right)\left(2^{n-1}-1\right)=2 \gamma(n)+2\left(\frac{2^{n+1}}{4}-1\right) \\
& \cdot\left(\frac{2^{n+1}}{4}\right)=2\left[2^{n+1}\right. \\
& \left.+2 \sum_{k=5}^{n}\left(2^{n-k}\right)\left(\frac{2^{k}}{4}-1\right)\left(\frac{2^{k}}{4}\right)\right]+2\left(\frac{2^{n+1}}{4}-1\right) \\
& \cdot\left(\frac{2^{n+1}}{4}\right)=2 \cdot 2^{n+1}+2 \\
& \cdot \sum_{k=5}^{n}\left(2^{n+1-k}\right)\left(\frac{2^{k}}{4}-1\right)\left(\frac{2^{k}}{4}\right)+2\left(\frac{2^{n+1}}{4}-1\right) \\
& \left(\frac{2^{n+1}}{4}\right)=2^{(n+1)+1} \\
& +2\left[\sum_{k=5}^{n}\left(2^{n+1-k}\right)\left(\frac{2^{k}}{4}-1\right)\left(\frac{2^{k}}{4}\right)\right. \\
& \left.+\left(2^{(n+1)-(n+1)}\right)\left(\frac{2^{n+1}}{4}-1\right)\left(\frac{2^{n+1}}{4}\right)\right]=2^{(n+1)+1} \\
& +2\left[\sum_{k=5}^{n+1}\left(2^{(n+1)-k}\right)\left(\frac{2^{k}}{4}-1\right)\left(\frac{2^{k}}{4}\right)\right] \text {. }
\end{aligned}
$$

The next theorem shows how effective the relaxation is.

Theorem 12 (see [8]). Let OPT be the optimal value of the MAXCUT problem and SDP be the optimal value of the semidefinite relaxation. Then

$$
1 \geq \frac{O P T}{S D P} \geq 0.87856 \cdots
$$

Theorem 12 guarantees that the optimal value of the MAXCUT is close to the optimal value of the semidefinite relaxation. From (4), we have

$$
\operatorname{AP}\left(\nu_{2}\left(Q_{n}\right)\right)=\left|E_{n}^{\prime}\right|-\operatorname{AP}\left(\operatorname{MC}\left(Q_{n}^{\prime}\right)\right),
$$

where $\left|E_{n}^{\prime}\right|$ is a number of potential crossing of 2-page drawing of $Q_{n} \cdot \operatorname{AP}\left(\nu_{2}\left(Q_{n}\right)\right)$ is an approximation of 2-page fixed linear crossing number of $Q_{n}$ and $\operatorname{AP}\left(\mathrm{MC}\left(Q_{n}^{\prime}\right)\right)$ is an approximation of $\mathrm{MC}\left(Q_{n}^{\prime}\right)$.

Corollary 13. Let $\operatorname{AP}\left(\nu_{2}\left(Q_{n}\right)\right)$ be an approximation of $v_{2}\left(Q_{n}\right)$. Then we have

$$
\operatorname{AP}\left(\nu_{2}\left(Q_{n}\right)\right) \leq v_{2}\left(Q_{n}\right) \leq k(n) \operatorname{AP}\left(v_{2}\left(Q_{n}\right)\right),
$$

where $k(n)$ is a computable quantity depending on $n$.
TABLE 1: The numerical results of the approximation of 2-page fixed linear crossing number of $Q_{n}, \operatorname{AP}\left(v_{2}\left(Q_{n}\right)\right)$ for $n=4,5,6$.

\begin{tabular}{cccc}
\hline$n$ & $\mathrm{AP}\left(\mathrm{MC}\left(Q_{n}^{\prime}\right)\right)$ & $\left|E_{n}^{\prime}\right|$ & $\mathrm{AP}\left(\nu_{2}\left(Q_{n}\right)\right)$ \\
\hline 4 & 35 & 40 & 5 \\
5 & 207 & 256 & 48 \\
6 & 1034 & 1344 & 310 \\
\hline
\end{tabular}

TABLE 2: The computable quantity $k(n)$, the bound of $v_{2}\left(Q_{n}\right)$, and the upper bound of $\operatorname{cr}\left(Q_{n}\right)$ for $n=4,5,6$.

\begin{tabular}{ccccc}
\hline$n$ & $k(n)$ & $\mathrm{AP}\left(\nu_{2}\left(Q_{n}\right)\right)$ & $k(n) \operatorname{AP}\left(\nu_{2}(G)\right)$ & $\begin{array}{c}\text { Upper bound } \\
\text { of } \operatorname{cr}\left(Q_{n}\right)\end{array}$ \\
\hline 4 & 1.91 & 5 & 9.55 & 8 \\
5 & 1.55 & 48 & 74.36 & 56 \\
6 & 1.43 & 310 & 444.42 & 352 \\
\hline
\end{tabular}

Proof. From (4), (18), and (19), we have

$$
\begin{aligned}
\frac{v_{2}\left(Q_{n}\right)}{\operatorname{AP}\left(v_{2}\left(Q_{n}\right)\right)} & =\frac{\left|E_{n}^{\prime}\right|-\operatorname{MC}\left(Q_{n}^{\prime}\right)}{\left|E_{n}^{\prime}\right|-\operatorname{AP}\left(\operatorname{MC}\left(Q_{n}^{\prime}\right)\right)} \\
& \leq \frac{\left|E_{n}^{\prime}\right|-0.87856 \operatorname{AP}\left(\operatorname{MC}\left(Q_{n}^{\prime}\right)\right)}{\left|E_{n}^{\prime}\right|-\operatorname{AP}\left(\operatorname{MC}\left(Q_{n}^{\prime}\right)\right)} .
\end{aligned}
$$

Let $k(n)=\left(\left|E_{n}^{\prime}\right|-0.87856 \mathrm{AP}\left(\mathrm{MC}\left(Q_{n}^{\prime}\right)\right)\right) /\left(\left|E_{n}^{\prime}\right|-\right.$ $\left.\operatorname{AP}\left(\operatorname{MC}\left(Q_{n}^{\prime}\right)\right)\right)$ be the computable quantity depending on $n$.

Then,

$$
\begin{aligned}
\frac{\nu_{2}\left(Q_{n}\right)}{\operatorname{AP}\left(\nu_{2}\left(Q_{n}\right)\right)} & \leq k(n) \\
v_{2}\left(Q_{n}\right) & \leq k(n) \cdot \operatorname{AP}\left(\nu_{2}\left(Q_{n}\right)\right) .
\end{aligned}
$$

Corollary 13 shows that the upper bound of $v_{2}\left(Q_{n}\right)$ is $k(n) \cdot \operatorname{AP}\left(\nu_{2}\left(Q_{n}\right)\right)$, where $k(n)$ is the computable quantity depending on $n$.

3.2. Experimental Results. In this section, we consider the hypercube graph $Q_{n}$ for $n=4,5,6$. Then, we give some examples for approximating the problems of the semidefinite relaxation in the form (8). We approximate this problem via MATLAB program together with an optimization toolbox called "SeDuMi." The SeDuMi is a package for solving optimization problems with linear, quadratic, and semidefinite constraints.

In Table 1 , the second column shows numerical results for the approximation of the MAXCUT on the associated conflict graph $Q_{n}^{\prime}$ by using the semidefinite relaxation. It is well known that this problem can be solved in a polynomial time. The third column displays the numbers of potential crossing of 2page drawing of $Q_{n}$ referring to our fixed vertex ordering that we evaluate from (14). Notice that this potential crossing of 2page drawing of $Q_{n}$ is the exact value. From (19), we calculate the approximation of 2-page fixed linear crossing number of $Q_{n}$ for $n=4,5,6$. The results are shown in the last column.

In Table 2, we present the lower bound of $v_{2}\left(Q_{n}\right)$, $\operatorname{AP}\left(\nu_{2}\left(Q_{n}\right)\right)$ and the upper bound of $\nu_{2}\left(Q_{n}\right), k(n) \operatorname{AP}\left(\nu_{2}(G)\right)$. 
The second column shows the values of $k(n)$ for $n=4,5,6$. We see that as $n$ get larger the values of $k(n)$ tend to decline continuously. It is interesting to study the behavior of $k(n)$ as $n \rightarrow \infty$. It does not surprise to see that our approximation is strictly larger than the upper bound of $\operatorname{cr}\left(Q_{n}\right)$ (14) since the latter one does not have a restriction that all vertices must be placed on a line. However, it is surprising to see that these numbers are not so different from each other.

\section{Concluding Remarks}

In this paper, given graph $G$, we show how the associating conflict graph $G^{\prime}$ is constructed. We recharacterize the problem of finding the crossing number of graph $G$ to the MAXCUT problem of $G^{\prime}$. We approximate the MAXCUT problem by the semidefinite relaxation which can be solved easily by a standard optimization package; in this case, we use SeDuMi 1.02. The numerical results show reasonable outcome. Clearly, another relaxation method can be explored. Moreover, it would be quite interesting to see the behavior of $k(n)$ as $n$ get larger. One can further study how to estimate $k(n)$ for a larger $n$.

\section{Conflicts of Interest}

The authors declare that there are no conflicts of interest regarding the publication of this paper.

\section{Acknowledgments}

The authors would like to thank the Thailand Research Fund under Project RTA5780007 and Chiang Mai University, Chiang Mai, Thailand, for the financial support.

\section{References}

[1] P. Erdös and R. K. Guy, "Crossing number problems," The American Mathematical Monthly, vol. 80, pp. 52-58, 1973.

[2] M. R. Garey and D. S. Johnson, "Crossing number is NP-complete," Society for Industrial and Applied Mathematics. Journal on Algebraic and Discrete Methods, vol. 4, no. 3, pp. 312-316, 1983.

[3] R. B. Eggleton and R. P. Guy, "The crossing number of the $n$ cube," Notices of the American Mathematical Society, vol. 17, p. $757,1970$.

[4] O. Sýkora and I. Vrto, "On crossing numbers of hypercubes and cube connected cycles," BIT Numerical Mathematics, vol. 33, no. 2, pp. 232-237, 1993.

[5] L. Faria, C. M. H. de Figueiredo, O. Sýkora, and I. Vrt'o, "An improved upper bound on the crossing number of the hypercube," Journal of Graph Theory, vol. 59, no. 2, pp. 145-161, 2008.

[6] E. de Klerk and D. V. Pasechnik, "Improved lower bounds for the 2-page crossing numbers of $K_{m, n}$ and $K_{n}$ via semidefinite programming," SIAM Journal on Optimization, vol. 22 , no. 2 , pp. 581-595, 2012.

[7] C. Buchheim and L. Zheng, "Fixed Linear Crossing Minimization by Reduction to The Maximum Cut Problem," in Computing and combinatorics, vol. 4112 of Lecture Notes in Computer Science, pp. 507-516, Springer, Berlin, Germany, 2006.
[8] A. Ben-Tal and A. Nemirovski, Lectures on Modern Convex Optimization: Analysis, Algorithms, and Engineering Applications, MOS-SIAM Series on Optimization, Society for Industrial and Applied Mathematics (SIAM), Pennsylvania, Pa, USA, 2001. 


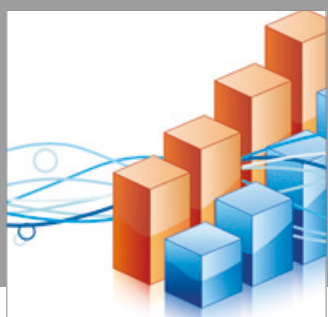

Advances in

Operations Research

vatersals

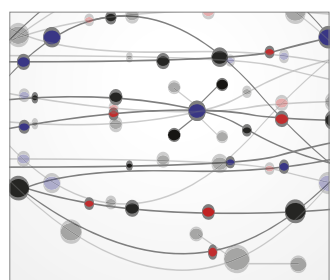

\section{The Scientific} World Journal
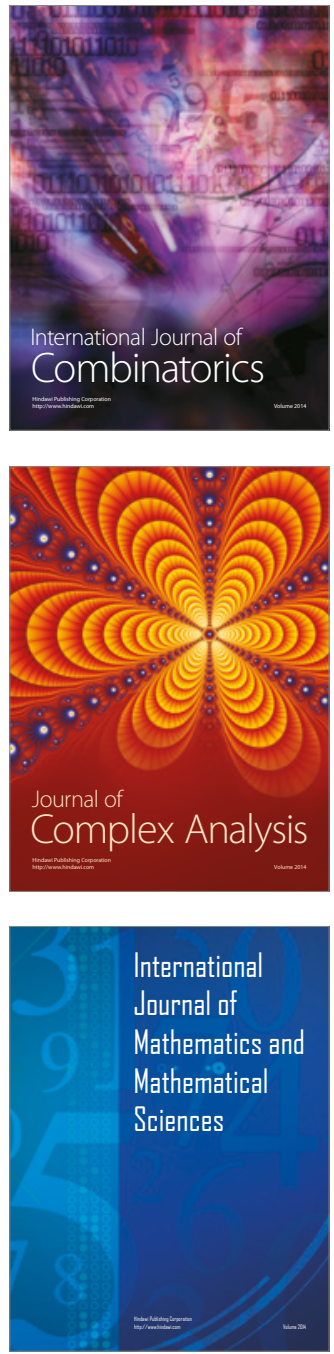
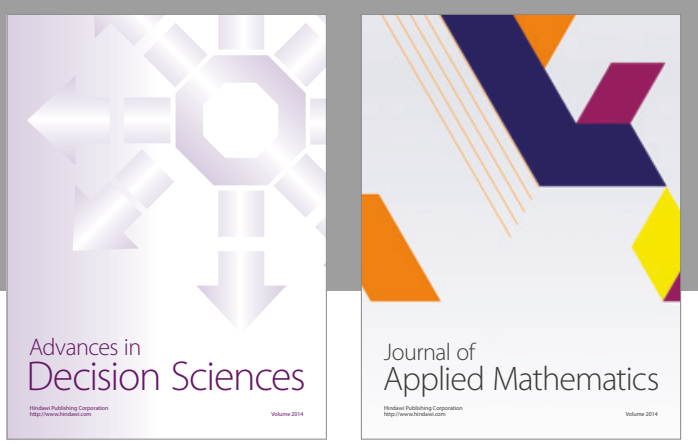

Algebra

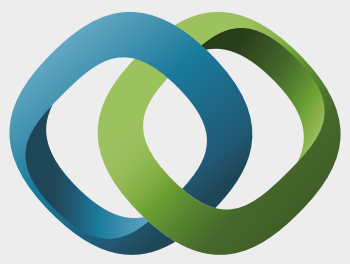

\section{Hindawi}

Submit your manuscripts at

https://www.hindawi.com
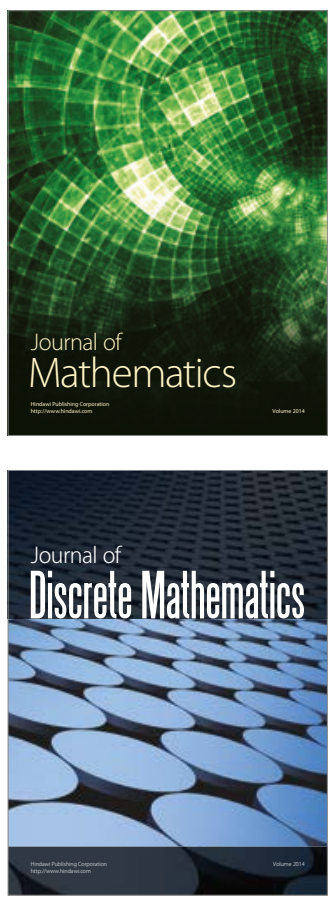

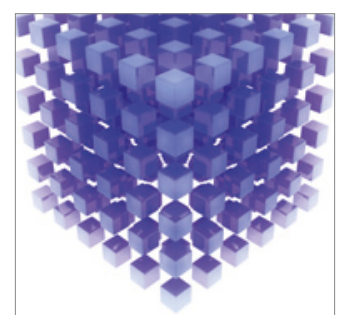

Mathematical Problems in Engineering
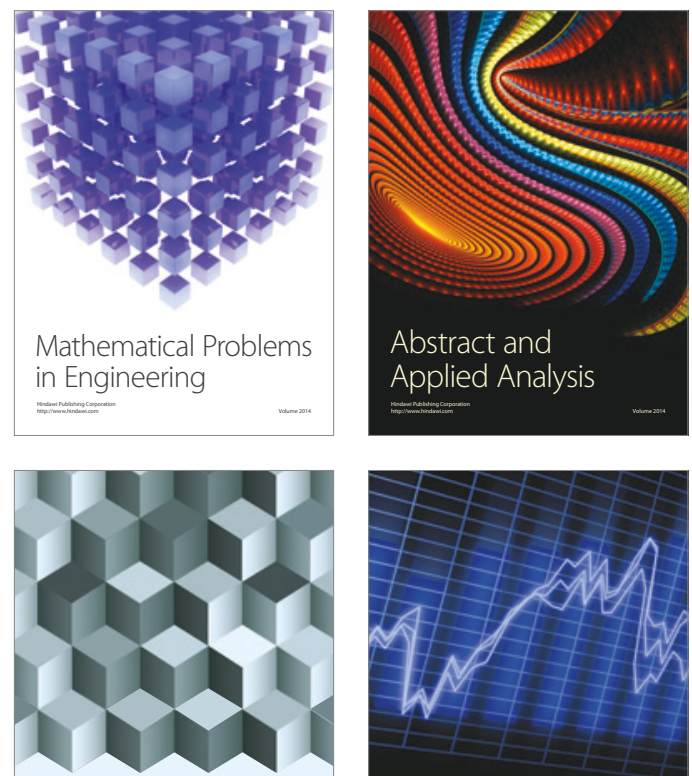

Journal of

Function Spaces

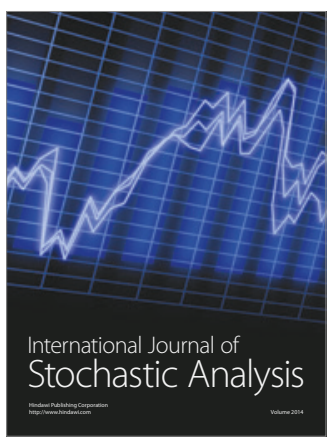

Probability and Statistics
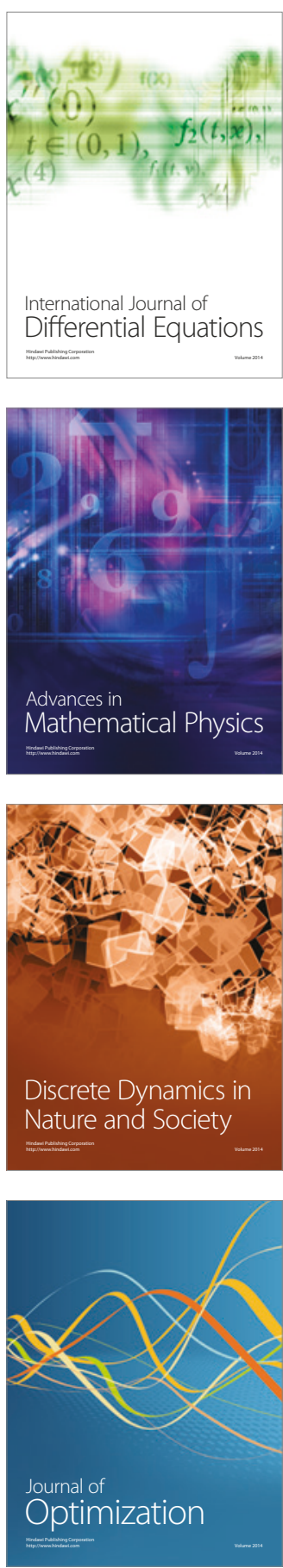